\title{
ĐỊNH VỊ ĐIỂM CHÍNH XÁC TÍCH HỌ̣P TRỊ ĐO GPS VÀ GLONASS
}

\author{
PGS. TS. NGUYẼ̃N NGỌC LÂU \\ Trường Đại học Bách khoa TP. Hồ Chí Minh
}

\section{Tóm tắt:}

Để nâng cao độ chính xác định vị điểm, chúng tôi đã nghiên cứu phương pháp xử lý tích hợp GPS và GLONASS sao cho có thể khắc phục sai số giữa hai hệ thống và sự khác nhau về sai số trị đo. Khi áp dụng phương pháp xử lý này cho 17 điểm IGS, độ chính xác hướng Đông và độ cao cải thiện so với GPS khoảng 11\% và $23 \%$. Với ca đo 60 phút, độ chính xác định vị có thể đạt được $2 \mathrm{~cm}$ mặt bằng và độ cao. Kết quả xử lý của chúng tồi tốt hơn phần mềm khác khoảng 1.5 lần ở thành phần mặt bằng và 1.3-2.0 lần ở thành phần độ cao.

\section{Giới thiệu}

Để nâng cao độ chính xác định vị điểm đơn (Precise Point Positioning-PPP), một trong những biện pháp hiệu quả nhất là xử lý tích hợp trị đo từ các hệ thống vệ tịnh định vị (Global Navigation Satellite Systems - GNSS) khác nhau hiện nay như GPS và GLONASS. Khi tích hợp, số lượng vệ tinh quan sát được nâng lên đáng kể làm gia tăng số trị đo thừa, dẫn đến cải thiện độ chính xác.

Việc tích hợp đòi hỏi giải quyết hàng loạt vấn đề liên quan sự khác nhau giữa các GNSS như hệ tọa độ và thời gian, bản lịch vệ tinh, độ chính xác trị đo, các nguồn sai số hệ thống, v.v... Trong nghiên cứu trước đây [5], chúng tôi đã chỉ ra rằng dùng bản lịch vệ tịnh của tổ chức IGS [3] có thể giải quyết vấn đề về hệ thống tọa độ và thời gian. Chỉ còn lại vấn đề quan trọng nhất cần giải quyết là sai số trị đo và sai số hệ thống giữa các GNSS (InterSystem Bias - ISB).

Trong bài báo này chúng tôi sẽ trình bày phương pháp khắc phục sai số giữa các hệ thống (ISB) ở mục 2. Khảo sát và chọn lựa mô hình sai số cho trị đo pha và mã của GPS và GLONASS ở mục 3 . Chúng tôi cũng chứng minh khả năng cải thiện độ chính xác khi tích hợp GPS+GLONASS ở mục 4.

\section{Phương pháp khắc phục ISB trong xử lý tích hợp GPS và GLONASS}

Tương tự như GPS, phương trình trị đo pha GLONASS trên 2 tần số từ máy thu i đến vệ tinh $\mathrm{k}$ ở thời điểm $\mathrm{t}$ có thể biểu diễn bằng các phương trình sau $[1,2]$

$$
\begin{aligned}
& L_{i, 1}^{k}(t)=\lambda_{1}^{k} \phi_{i, 1}^{k}(t)=\rho_{i}^{k}(t)+c\left[d t_{i}(t)-d T^{k}(t)\right]-I_{i, 1}^{k}(t)+T_{i}^{k}(t)+\lambda_{1}^{k} N_{i, 1}^{k}+\lambda_{1}^{k}\left[\phi_{i, 1}\left(t_{0}\right)-\phi_{1}^{k}\left(t_{0}\right)\right]+e_{i, 1}^{k}(t) \\
& L_{i, 2}^{k}(t)=\lambda_{2}^{k} \phi_{i, 2}^{k}(t)=\rho_{i}^{k}(t)+c\left[d t_{i}(t)-d T^{k}(t)\right]-I_{i, 2}^{k}(t)+T_{i}^{k}(t)+\lambda_{2}^{k} N_{i, 2}^{k}+\lambda_{2}^{k}\left[\phi_{i, 2}\left(t_{0}\right)-\phi_{1}^{k}\left(t_{0}\right)\right]+e_{i, 2}^{k}(t)
\end{aligned}
$$

Người phản biện: PGS. TSKH. Hà Minh Hòa 
Trong đó:

$\rho_{i}^{k}(t)$ là khoảng cách hình học từ máy thu i đến vệ tinh $\mathrm{k}$ ở thời điểm $\mathrm{t}$

$f_{1}^{k}$ và $\lambda_{1}^{k}$ là tần số và bước sóng sóng tải $\mathrm{L}_{1}$ của vệ tinh $\mathrm{k}$

$d t_{i}(t)$ và $d T^{k}(t)$ là sai số đồng hồ máy thu $\mathrm{i}$ và vệ tinh $\mathrm{k}$ ở thời điểm $\mathrm{t}$

$I_{i, 1}^{k}(t)$ là độ trễ điện ly trên tần số $\mathrm{L}_{1}$ ở thời điểm $\mathrm{t}$

$T_{i}^{k}(t)$ là độ trễ đối lưu ở thời điểm $\mathrm{t}$

$N_{i, 1}^{k}$ là tham số đa trị trên tần số $\mathrm{L}_{1}$ ở thời điểm $\mathrm{t}$

$\phi_{i, 1}\left(t_{0}\right)$ và $\phi_{1}^{k}\left(t_{0}\right)$ là pha ban đầu của máy thu i và vệ tinh $\mathrm{k}$

$\varepsilon_{i, 1}^{k}(t)$ bao gồm sai số do đa đường và độ nhiễu đo đạc trên tần số $\mathrm{L}_{1}$ ở thời điểm $\mathrm{t}$

Để khử đi ảnh hưởng của tầng điện ly có mặt trong các phương trình, người ta thường sử dụng trị đo kết hợp $\mathrm{L}_{3}$ có dạng sau:

$$
L_{i, 3}^{k}(t)=\alpha_{1} L_{i, 1}^{k}(t)-\alpha_{2} L_{i, 2}^{k}(t)=\rho_{i}^{k}(t)+c\left\lfloor d t_{i}(t)-d T^{k}(t)\right\rfloor+T_{i}^{k}(t)+b_{i, 3}^{k}+e_{i, 3}^{k}(t)
$$

Trong đó: $\quad \alpha_{1}=\frac{\left(f_{1}^{k}\right)^{2}}{\left(f_{1}^{k}\right)^{2}-\left(f_{2}^{k}\right)^{2}}$ và $\quad \alpha_{2}=\frac{\left(f_{2}^{k}\right)^{2}}{\left(f_{1}^{k}\right)^{2}-\left(f_{2}^{k}\right)^{2}}$

Các vệ tinh GLONASS thỏa mãn điều kiện $\frac{f_{2}^{k}}{f_{1}^{k}}=\frac{7}{9}$ [3]. Do đó dễ dàng chứng minh $\alpha_{1}=\frac{1}{1-49 / 81}=2.53125$ và $\alpha_{2}=\frac{1}{81 / 49-1}=1.5313$ không phụ thuộc vào vệ tinh

và $b_{i, 3}^{k}=\alpha_{1} \lambda_{1}^{k} N_{i, 1}^{k}-\alpha_{2} \lambda_{2}^{k} N_{i, 2}^{k}+\alpha_{1} \lambda_{1}^{k}\left[\phi_{i, 1}\left(t_{0}\right)-\phi_{1}^{k}\left(t_{0}\right)\right]-\alpha_{2} \lambda_{2}^{k}\left[\phi_{i, 2}\left(t_{0}\right)-\phi_{2}^{k}\left(t_{0}\right)\right]$ là sai số hệ thống không phụ thuộc thời gian.

Để xử lý kết hợp với GPS, theo [5], chúng ta cần thêm sai số ISB vào phương trình (3)

$$
\left.L_{i, 3}^{k}(t)=\alpha_{1} L_{i, 1}^{k}(t)-\alpha_{2} L_{i, 2}^{k}(t)=\rho_{i}^{k}(t)+c \mid d t_{i}(t)-d T^{k}(t)\right]+T_{i}^{k}(t)+b_{i, 3}^{k}+c . I S B(t)+e_{i, 3}^{k}(t)
$$

Tương tự chúng ta cũng có phương trình cho trị đo giả cự ly

$$
P_{i, 3}^{k}(t)=\alpha_{1} P_{i, 1}^{k}(t)-\alpha_{2} P_{i, 2}^{k}(t)=\rho_{i}^{k}(t)+c\left[d t_{i}(t)-d T^{k}(t)\right]+T_{i}^{k}(t)+c . I S B(t)+\bar{e}_{i, 3}^{k}(t)
$$

Các phương trình (4) vẫn còn chứa đựng các sai số đồng hồ và sai số ISB. Để khử chúng đi người ta thường thành lập các phương trình ở dạng hiệu đơn giữa các vệ tinh GLONASS k và I như sau:

$$
\begin{gathered}
L_{i, 3}^{k l}(t)=\rho_{i}^{k l}(t)-c . d T^{k l}(t)+T_{i}^{k l}(t)+b_{i, 3}^{k l}+e_{i, 3}^{k l}(t) \\
P_{i, 3}^{k l}(t)=\rho_{i}^{k l}(t)-c . d T^{k l}(t)+T_{i}^{k l}(t)+\bar{e}_{i, 3}^{k l}(t)
\end{gathered}
$$

Các phương trình (5) đã loại bỏ hoàn toàn sai số đồng hồ máy thu và ISB, chỉ còn lại hiệu sai số đồng hồ vệ tinh, độ trễ đối lưu và các nguồn sai số hệ thống khác. 
Với việc xử lý kết hợp GPS và GLONASS trên cơ sở sử dụng các phương trình (5), chúng ta có hai sơ đồ kết hợp sau:

- Xử lý riêng biệt GPS và GLONASS rồi sau đó kết hợp nghiệm với nhau.

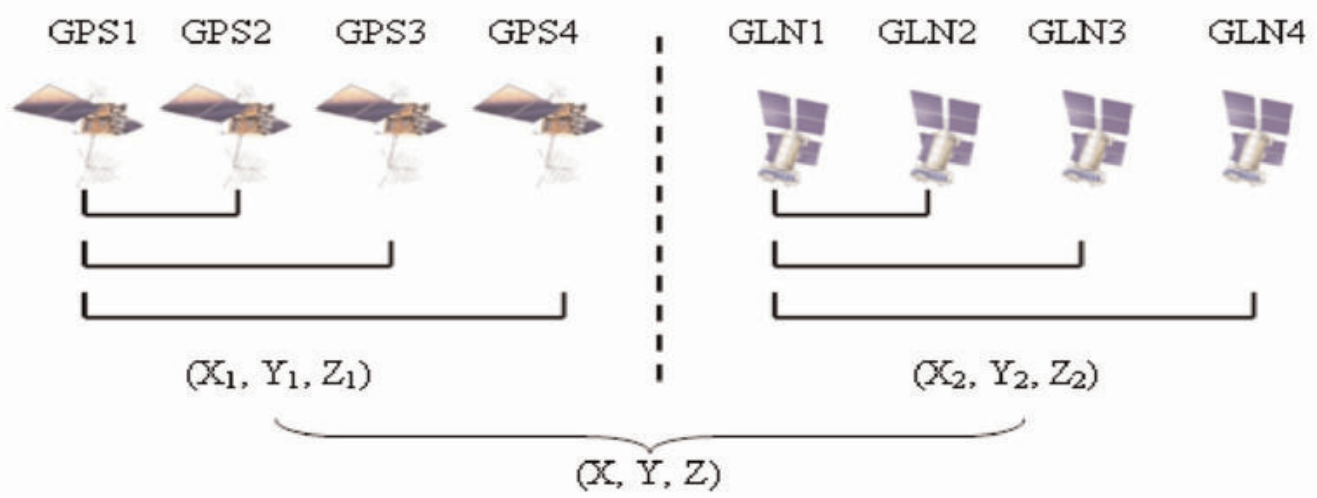

Hình 1: Kết hợp nghiệm xử lý riêng ở hai hệ thống

Vì xử lý riêng nên phương pháp này có ưu điểm là không cần quan tâm đến sự khác nhau về độ chính xác giữa trị đo GPS và GLONASS. Chương trình xử lý trị đo GPS có thể chuyển đổi sang xử lý GLONASS chỉ với vài sự thay đổi nhỏ. Nhược điểm của phương pháp là khi số lượng vệ tinh của từng hệ thống nhỏ hơn 4 thì không thể cho nghiệm tương ứng.

- Xử lý cùng lúc các trị đo GPS-GPS và GLONASS-GLONASS

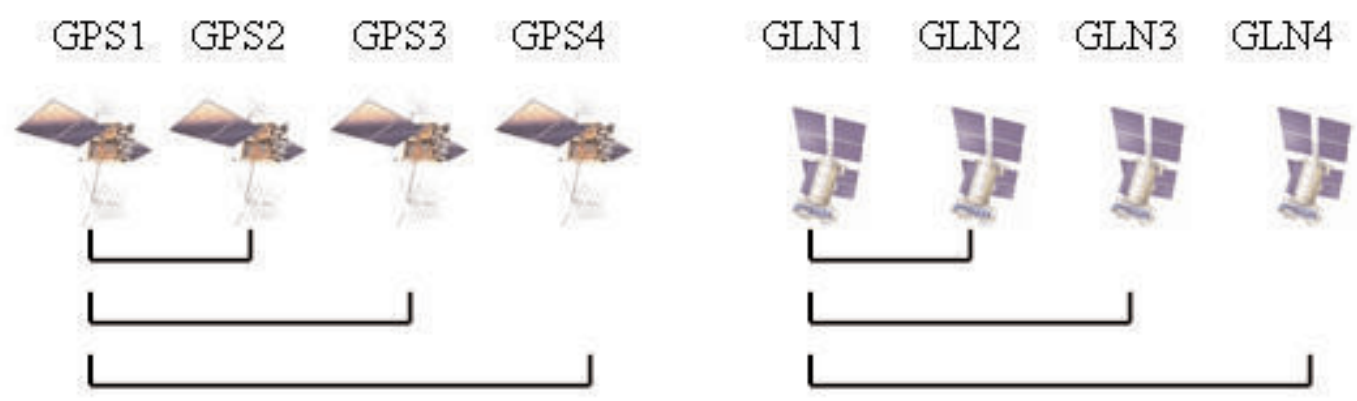

Hình 2: Xủ̉ lý chung trị đo GPS-GPS và GLONASS-GLONASS

Chỉ cần số vệ tinh của một hệ thống 3 , hệ thống kia 2 thì cách thức này vẫn cho nghiệm. Tuy nhiên vì xử lý đồng thời các trị đo hiệu GPS-GPS và GLONASS-GLONASS, nên cần phải giải quyết vấn đề khác biệt về độ chính xác giữa hai loại trị đo. Việc lập trình cũng tương đối phức tạp do phải thành lập hiệu trong từng hệ thống. Ngoài ra nếu hệ thống nào đó chỉ có một vệ tinh, thì trị đo tương ứng cũng phải bỏ qua một cách lãng phí.

Nếu vệ tinh k là GPS, vệ tinh I là GLONASS, thì phương trình (4) sẽ có dạng

$$
\mathrm{L}_{\mathrm{i}, 3}^{\mathrm{kl}}(\mathrm{t})=\rho_{\mathrm{i}}^{\mathrm{kl}}(\mathrm{t})-\mathrm{c} \cdot \mathrm{dT}^{\mathrm{kl}}(\mathrm{t})+\mathrm{T}_{\mathrm{i}}^{\mathrm{kl}}(\mathrm{t})+\mathrm{b}_{\mathrm{i}, 3}^{\mathrm{kl}}-\mathrm{c} \cdot \operatorname{ISB}(\mathrm{t})+\mathrm{e}_{\mathrm{i}, 3}^{\mathrm{kl}}(\mathrm{t})
$$




$$
P_{i, 3}^{k l}(t)=\rho_{i}^{k l}(t)-c . d T^{k l}(t)+T_{i}^{k l}(t)-c . I S B(t)+\bar{e}_{i, 3}^{k l}(t)
$$

Khi xử lý kết hợp GPS và GLONASS trên cơ sở sử dụng các phương trình (6), chúng ta có sơ đồ kết hợp sau:

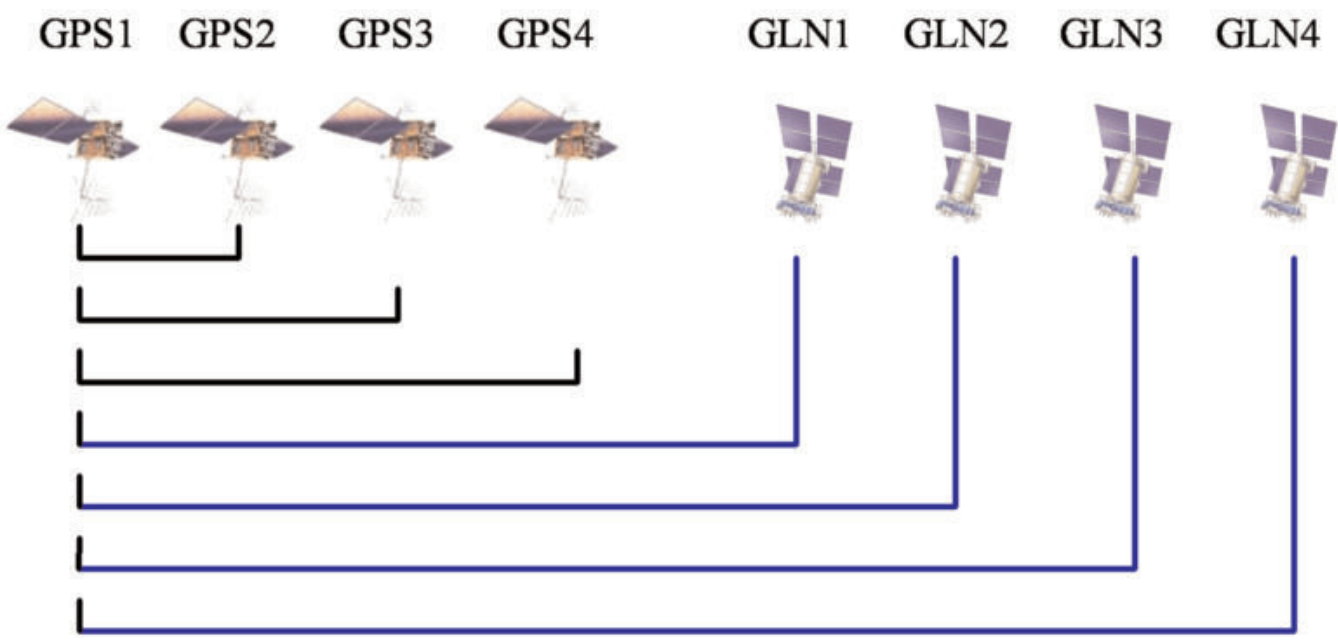

Hình 3: Xử lý đồng thời trị đo GPS-GPS và GPS-GLONASS

Đây là cách kết hợp cho số lượng trị đo nhiều nhất, khai thác được trị đo khi chỉ có một vệ tinh trong hệ thống. Với một chương trình xử lý PPP dùng trị đo GPS đã có thì việc mở rộng để xử lý hỗn hợp GPS+GLONASS theo mô hình trên sẽ không quá phức tạp. Mặt khác vì trị đo GPS chính xác hơn GLONASS, nên trị đo hiệu GPS-GLONASS sẽ chính xác hơn GLONASS-GLONASS, cho phép chúng ta cải thiện độ chính xác định vị.

Trong nghiên cứu này, chúng tôi sẽ chọn phương án trên để xử lý tích hợp GPS và GLONASS. Sai số ISB được xem là ẩn số bổ sung, được khảo sát cùng với các ẩn số tọa độ. Vì tham số này biến động theo thời gian [7], chúng tôi xử lý nó theo bước ngẫu nhiên (random walk) với tần suất cập nhật 15 phút/1 lần.

\section{Xây dựng mô hình sai số cho các trị đo GPS và GLONASS}

Để khảo sát mô hình sai số các trị đo, chúng tôi dùng dữ liệu của 17 trạm đo IGS vào ngày 26-04-2012. Máy thu tại các trạm đo này đều thu được tín hiệu GPS và GLONASS. Tọa độ của chúng được cho trong hệ ITRF2008 với độ chính xác vài $\mathrm{mm}$. Để cho kết quả tương đối khách quan, chúng tôi cố gắng chọn nhiều loại máy thu khác nhau (xem bảng 1). Dữ liệu đo của các trạm này ở dạng RINEX, được sửa chữa trượt chu kỳ pha theo thuật toán Blewitt [6]. (Xem bảng 1)

Kết quả khảo sát cho thấy sai số trị đo pha $\mathrm{L}_{3}$ và trị đo mã $\mathrm{P}_{3}$ của GPS được xấp xỉ bằng công thức:

$$
\sigma_{\text {phase }}^{G P S}=0.0046\left(0.1+\frac{1}{\sin \varepsilon}\right) \quad ; \quad \sigma_{\text {code }}^{G P S}=0.3203\left(0.1+\frac{1}{\sin \varepsilon}\right)
$$

Sai số trị đo pha $\mathrm{L}_{3}$ và trị đo mã $\mathrm{P}_{3}$ của GLONASS được xấp xỉ bằng công thức: 
Bảng 1: Tọa độ ITRF2008 của một số trạm đo IGS ngày 26-04-2012

\begin{tabular}{|c|c|c|c|c|c|}
\hline STT & $\begin{array}{c}\text { Trạm } \\
\text { đo }\end{array}$ & $\mathbf{x}$ & $\mathbf{Y}$ & z & Receiver/antenna \\
\hline 1 & ALIC & -4052052.4375 & 4212836.0313 & $3-2545105.0041$ & $\begin{array}{c}\text { LEICA } \\
\text { GRX1200GGPRO/LEIAR25.R3 }\end{array}$ \\
\hline 2 & AUCK & -5105681.3473 & 461564.0137 & -3782181.2441 & TRIMBLE NETR9/TRM55971.00 \\
\hline 3 & BAKO & -1836969.3060 & 6065617.0150 & -716257.8760 & $\begin{array}{c}\text { LEICA } \\
\text { GRX1200GGPRO/LEIAT504GG }\end{array}$ \\
\hline 4 & CAS1 & -901776.1423 & 2409383.2805 & $5 \mid-5816748.4848$ & $\begin{array}{c}\text { LEICA } \\
\text { GRX1200GGPRO/AOAD/M_T }\end{array}$ \\
\hline 5 & CEDU & -3753472.8740 & 3912741.0142 & -3347960.0829 & TRIMBLE NETR8/AOAD/M_T \\
\hline 6 & DARW & -4091359.3326 & 4684606.5294 & -1408579.5750 & \begin{tabular}{|c} 
LEICA \\
GRX1200GGPRO/ASH700936D_M
\end{tabular} \\
\hline 7 & DAV1 & 486854.5704 & 2285099.2198 & -5914955.6939 & $\begin{array}{c}\text { LEICA } \\
\text { GRX1200GGPRO/LEIAR25.R3 }\end{array}$ \\
\hline 8 & GUUG & -5070465.2010 & 3576460.2796 & 1472093.8149 & TRIMBLE NETR5/TRM55971.00 \\
\hline 9 & HOB2 & -3950071.9619 & 2522415.3118 & \begin{tabular}{l|l}
3 & -4311637.7284
\end{tabular} & $\begin{array}{c}\text { LEICA } \\
\text { GRX1200GGPRO/AOAD/M_T }\end{array}$ \\
\hline 10 & LHAZ & -106941.7816 & 5549269.8267 & 3139215.1117 & TPS E_GGD/ASH701941.B \\
\hline 11 & MAC1 & -1508023.014 & 6195576.603 & 148799.362 & LEICA GRX1200+GNSS/AOAD/M_T \\
\hline 12 & NTUS & -1508023.0140 & 6195576.6030 & 148799.3620 & $\begin{array}{c}\text { LEICA } \\
\text { GRX1200GGPRO/LEIAT504GG }\end{array}$ \\
\hline 13 & TIXI & -1264873.3403 & 1569455.7703 & \begin{tabular}{l|l}
36031003.4087
\end{tabular} & JPS EGGDT/TPSCR3_GGD \\
\hline 14 & TOW2 & -5054583.1672 & 3275504.2328 & $3-2091538.8891$ & $\begin{array}{l}\text { LEICA } \\
\text { GRX1200GGPRO/LEIAR25.R3 }\end{array}$ \\
\hline 15 & URUM & 193030.4017 & 4606851.3022 & 4393311.5127 & TPS NETG3/TPSCR3_GGD \\
\hline 16 & WUHN & -2267749.6575 & 5009154.2355 & \begin{tabular}{|l|l|}
3221290.6291
\end{tabular} & TRIMBLE NETR8/TRM59800.00 \\
\hline 17 & XMIS & -1696344.4400 & 6039590.0150 & -1149275.5050 & $\begin{array}{c}\text { LEICA } \\
\text { GRX1200GGPRO/ASH1945C_M }\end{array}$ \\
\hline
\end{tabular}




$$
\sigma_{\text {phase }}^{G L N}=k_{1} \cdot \sigma_{\text {phase }}^{G P S} \quad \text { và } \quad \sigma_{\text {code }}^{G L N}=k_{2} \cdot \sigma_{\text {code }}^{G P S}
$$

Trong đó

$$
\mathrm{k}_{1}=\sigma_{\mathrm{phuse}}^{\mathrm{GLN}} / \sigma_{\text {phase }}^{\mathrm{GPS}}=1.2 ; \mathrm{k}_{2}=\sigma_{\text {code }}^{\mathrm{GLH}} / \sigma_{\text {code }}^{\mathrm{GPS}}=3.5
$$

Điều này chỉ ra rằng trị đo pha GPS chính xác hơn GLONASS 1.2 lần. Nhưng trị đo mã GLONASS lại kém chính xác hơn GPS đến 3.5 lần.

\section{Kết quả thử nghiệm và phân tích}

Chúng tôi áp dụng phương pháp xử lý tích hợp đã trình bày ở mục 2 và mô hình sai số các trị đo ở mục 3 để viết ra phần mềm định vị điểm chính xác PPPC. Để xem xét mức độ cải thiện khi tích hợp GPS và GLONASS, chúng tôi dùng PPPC xử lý tọa độ của 17 điểm IGS ở bảng 1, rồi so sánh với tọa độ chính xác của nó. Đối với mỗi điểm, chúng tôi chia dữ liệu $24 \mathrm{~h}$ thành các phân đoạn $0.5 \mathrm{~h}, 1,2,3,4,6$, 12, 24h và xử lý riêng biệt theo 3 phương án:

- Chỉ dùng trị đo GPS

- Chỉ dùng trị đo GLONASS

- Dùng trị đo GPS và GLONASS

Kết quả so sánh được trình bày ở hình 4 cho thấy độ chính xác định vị luôn tăng theo chiều dài của ca đo. Cùng một ca đo, GLONASS cho kết quả kém hơn GPS và GPS+GLONASS. Sự cải thiện của GPS+GLONASS so với GPS rõ nét nhất là ở thành phần hướng Đông $(\sim 11 \%)$ và độ cao ( 23\%). Trong khi ở thành phần hướng Bắc, GPS lại cho kết quả tốt hơn chút ít so với GPS+GLONASS. (Xem hinh 4)

Để đánh giá độ chính xác của PPPC, chúng tôi so sánh kết quả xử lý 24h với một số các dịch vụ định vị điểm chính xác có xử lý tích hợp GPS và GLONASS, bao gồm:

- Phần mềm magicGNSS phát triển bởi GMV tại trang web http://magicgnss.gmv.com/

- Phần mềm CSRS-PPP được phát triển bởi Cục Đo đạc thuộc Bộ Tài nguyên Canada tại trang web http://www.geod.nrcan.gc.ca/index_e.php

Độ lệch tọa độ giữa kết quả xử lý từ ba phần mềm so với tọa độ chính xác của 17 điểm IGS được tóm tắt ở bảng 2 . Theo đó độ chính xác định vị của CSRS-PPP và magicGNSS tương đương nhau với $11 \mathrm{~mm}$ ở thành phần mặt bằng và $12-19 \mathrm{~mm}$ ở thành phần độ cao. Tức là độ chính xác độ cao kém hơn mặt bằng từ 1.5 đến 2 lần. PPPC cho độ chính xác mặt bằng $7 \mathrm{~mm}$ và độ cao $9.4 \mathrm{~mm}$, tốt hơn các phần mềm kia 1.5 lần ở mặt bằng và 1.32.0 lần ở thành phần độ cao. (Xem bảng 2 ) 

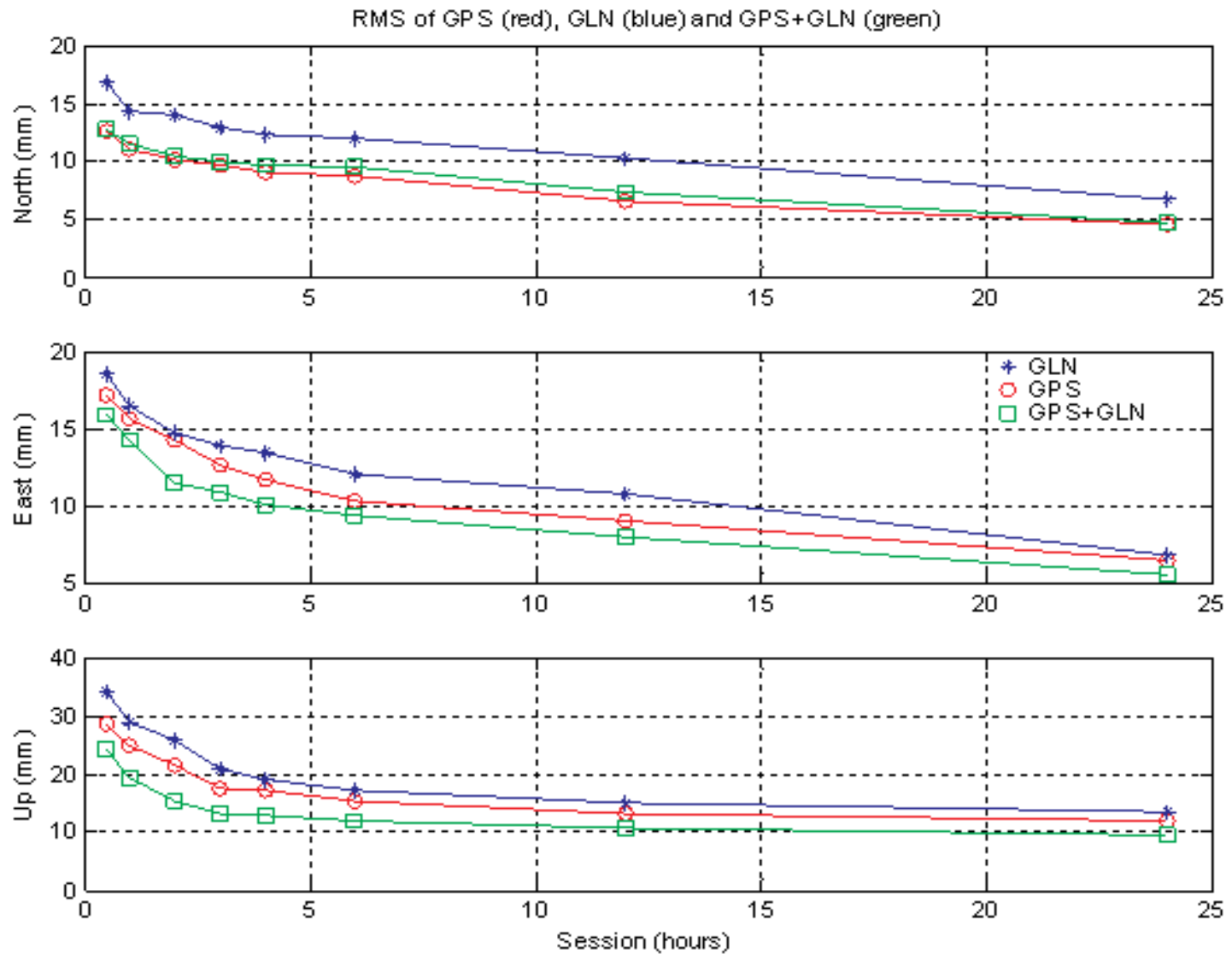

Hình 4: Độ chính xác định vị theo chiều dài ca đo

\section{Kết luận}

Chúng tôi đã trình bày phương pháp xử lý tích hợp trị đo GPS và GLONASS để khắc phục sai số hệ thống ISB và sự khác nhau về sai số trị đo. Dùng phần mềm PPPC do chúng tôi viết ra để xử lý dữ liệu đo của 17 trạm IGS. Kết quả xử lý cho thấy:

Độ chính xác khi xử lý trị đo GPS và GLONASS tốt hơn GPS chủ yếu ở thành phần hướng Đông và độ cao với mức độ cải thiện trung bình là $11 \%$ và $23 \%$. Với ca đo 60 phút, độ chính xác định vị có thể đạt được $2 \mathrm{~cm}$ mặt bằng lẫn độ cao.

PPPC cho độ chính xác tốt hơn các phần mềm CSRS-PPP và magicGNSS khoảng 1.5 lần ở thành phần mặt bằng và 1.3-2.0 lần ở thành phần độ cao.

Nghiên cứu này được tài trợ bởi Đại học Quốc gia Thành phố Hồ Chí Minh (VNU-HCM) trong đề tài mã số B2012-20-33.O 
Bảng 2: So sánh kết quả định vị của PPPC và các phần mềm khác

\begin{tabular}{|c|c|c|c|c|c|}
\hline STT & Trạm đo & North $(\mathrm{mm})$ & East (mm) & Up (mm) & Phần mềm \\
\hline & \multirow{3}{*}{ ALIC } & -2.7 & +4.1 & +3.6 & CSRS-PPP \\
\hline & & $\begin{array}{l}-1.3 \\
\end{array}$ & +13.2 & -20.9 & magicGNSS \\
\hline & & +3.2 & +7.2 & -5.9 & PPPC \\
\hline & \multirow{3}{*}{ AUCK } & -11.3 & -0.6 & +18.1 & CSRS-PPP \\
\hline & & -7.1 & +10.4 & -16.0 & magicGNSS \\
\hline & & -1.2 & -0.5 & -0.2 & PPPC \\
\hline & \multirow{3}{*}{ BAKO } & -4.1 & -18.7 & +34.7 & CSRS-PPP \\
\hline & & +5.6 & -6.4 & +2.7 & magicGNSS \\
\hline & & +0.5 & -7.6 & -3.0 & PPPC \\
\hline & \multirow{3}{*}{ CASI } & +0.1 & -6.7 & +13.4 & CSRS-PPP \\
\hline & & -3.6 & -1.9 & +20.0 & magicGNSS \\
\hline & & +3.4 & -3.9 & -2.0 & PPPC \\
\hline & \multirow{3}{*}{ CEDU } & -3.6 & -0.4 & +9.6 & CSRS-PPP \\
\hline & & -4.7 & +0.2 & -5.6 & magicGNSS \\
\hline & & +2.8 & +0.7 & +2.0 & PPPC \\
\hline & \multirow{3}{*}{ DARW } & -11.8 & -3.4 & +29.1 & CSRS-PPP \\
\hline & & -13.4 & +7.8 & -5.8 & magicGNSS \\
\hline & & -0.8 & +4.0 & -3.1 & PPPC \\
\hline & \multirow{3}{*}{ DAVI } & -5.7 & -4.6 & +29.0 & CSRS-PPP \\
\hline & & -1.4 & -7.0 & +15.5 & magicGNSS \\
\hline & & -3.2 & -5.3 & +1.6 & PPPC \\
\hline & \multirow{3}{*}{ GUUG } & -8.3 & +9.3 & -3.7 & CSRS-PPP \\
\hline & & -2.1 & +18.0 & -14.7 & magicGNSS \\
\hline & & -2.8 & +11.2 & -23.1 & PPPC \\
\hline & \multirow{3}{*}{ HOB2 } & -8.8 & -2.9 & +7.6 & CSRS-PPP \\
\hline & & -10.2 & +0.3 & -1.9 & magicGNSS \\
\hline & & -2.7 & +0.9 & -16.8 & PPPC \\
\hline & \multirow{3}{*}{ LHAZ } & +8.5 & -4.6 & +3.9 & CSRS-PPP \\
\hline & & -1.8 & -0.4 & +1.7 & magicGNSS \\
\hline & & -2.0 & -0.1 & +6.5 & PPPC \\
\hline & \multirow{3}{*}{ MAC1 } & -6.4 & -9.7 & +31.8 & CSRS-PPP \\
\hline & & -14.4 & -4.9 & +6.9 & magicGNSS \\
\hline & & -1.9 & -8.2 & +6.2 & PPPC \\
\hline & \multirow{3}{*}{ NTUS } & +13.7 & +12.2 & +13.6 & CSRS-PPP \\
\hline & & +8.0 & +7.3 & -19.5 & magicGNSS \\
\hline & & +10.5 & +9.3 & +3.3 & PPPC \\
\hline & \multirow{3}{*}{ TIXI } & +6.4 & -4.3 & +19.3 & CSRS-PPP \\
\hline & & +9.6 & -6.3 & -0.3 & magicGNSS \\
\hline & & +1.5 & -7.1 & -10.2 & PPPC \\
\hline & \multirow{3}{*}{ TOW2 } & +2.2 & +0.3 & +0.0 & CSRS-PPP \\
\hline & & +5.8 & +9.4 & -18.7 & magicGNSS \\
\hline & & +9.9 & +1.3 & -16.4 & PPPC \\
\hline & \multirow{3}{*}{ URUM } & +2.1 & +2.0 & +12.7 & CSRS-PPP \\
\hline & & +2.8 & +7.1 & -0.5 & magicGNSS \\
\hline & & -5.2 & +1.9 & -3.4 & PPPC \\
\hline & \multirow{3}{*}{ WUHN } & -1.0 & +2.1 & -10.3 & CSRS-PPP \\
\hline & & +1.9 & +5.7 & -8.2 & magicGNSS \\
\hline & & +2.5 & +2.1 & -8.6 & PPPC \\
\hline & \multirow{3}{*}{ XMIS } & -3.5 & -10.9 & +25.0 & CSRS-PPP \\
\hline & & +4.7 & -6.7 & +2.4 & magicGNSS \\
\hline & & +7.8 & -1.3 & +8.4 & PPPC \\
\hline & \multirow{3}{*}{ RMS } & 8.1 & 7.5 & 18.9 & CSRS-PPP \\
\hline & & 7.2 & 8.0 & 12.1 & magicGNSS \\
\hline & & 4.7 & 5.5 & 9.4 & PPPC \\
\hline
\end{tabular}




\section{Tài liệu tham khảo}

[1]. Teunissen, P.J.G., and A. Kleusberg, (1996), "GPS Observations and Positioning Concepts", in GPS for Geodesy, edited by A. Kleusberg and P. J. G. Teunissen, SpringerVerlag, pp. 175-217.

[2]. Heinz Habrich, (1999), "Geodetic application of the global navigation satellite system (GLONASS) and of GLONASS/GPS combination", University of Bern, 51pp.

[3]. GLONASS, (2002), "Interface Control Document version 5.0", coordinate scientific information center.

[4]. International GNSS Service (IGS) có địa chỉ trang web http://igscb.jpl.nasa.gov/.

[5]. Nguyễn Ngọc Lâu, (2012), "Nghiên cứu sử dụng sản phẩm bản lịch và số hiệu chỉnh đồng hồ vệ tinh GLONASS cho định vị điểm đơn chính xác tích hợp GPS và GLONASS", Tuyển tập báo cáo Hội nghị Khoa học và Công nghệ "Trắc địa và Bản đồ vì sự nghiệp Tài nguyên và Môi trường" do Viện Khoa học Đo đạc và Bản đồ phối hợp với Hội Trắc địa-Bản đồ-Viễn thám tổ chức tại Hà Nội, 10-2012, pp. 17-26.

[6]. Nguyễn Ngọc Lâu và Nguyễn Văn Tuấn, (2013), "Khảo sát thuật toán Blewitt trong phát hiện trượt chu kỳ trên trị đo pha GPS và GLONASS", Tạp chí Khoa học Đo đạc và Bản đồ, Số 15-3/2013.

[7]. S. Schaer, R. Dach, (2010), "Biases in GNSS analysis", IGS Workshop, Newcastle, England, 28 June - 2 July 2010.0

\section{Summary}

POINT PRECISE POSITIONING USING GPS AND GLONASS MEASUREMENTS

Assoc. Prof. Dr. Nguyen Ngoc Lau

Hochiminh City University of Technology

To improve accuracy of precise point positioning, we research a method for processing mixed GPS and GLONASS measurements. This method is able to overcome inter-system biases and measurement errors. Processing 17 ISG stations, the results show that North and Up accuracies are improved about $11 \%$ and $23 \%$ compared with GPS only. For 60 minutes session, the positional accuracy can be $2 \mathrm{~cm}$ in the horizontal and the vertical component. Our results are better than other software packages about 1.5 times in the horizontal and 1.3-2.0 times in the vertical component. $O$

Ngày nhận bài: 10/12/2012. 